\title{
Loss of Atypical Chemokine Receptors Expression Promotes Lymph Node Metastasis of Colorectal Cancer
}

\section{Haitao Duan \\ Yao Huang \\ Dalian Medical University \\ Caihua Zhang \\ Dalian Medical University \\ Juanjuan Cui}

The First Affiliated Hospital of Dalian medical university

The first affiliated hospital of Anhui medical university

\section{Lianying Guo}

Dalian Medical University

\section{Ting Zhang}

Dalian Medical University

\section{Lijun Yu}

Dalian Medical University

\section{Dongnin Li}

Dalian Medical University

\section{Lu Kong}

Dalian Medical University

\section{Pin Liang ( $\nabla$ pinliang124@163.com )}

The First Affiliated Hospital of Dalian medical university

\section{Research}

Keywords: atypical chemokine receptor, colorectal cancer, metastasis, DARC, D6, CCX-CKR, survival analysis

Posted Date: January 21st, 2021

DOI: https://doi.org/10.21203/rs.3.rs-150865/v1

License: (c) (i) This work is licensed under a Creative Commons Attribution 4.0 International License. Read Full License 


\section{Abstract}

Background: Atypical chemokine receptors (ACKRs), including DARC, D6 and CCX-CKR, hold very critical roles in cancer invasion and metastasis. The objective of the present study was to determine preliminarily the predictive value of ACKRs in colorectal cancer (CRC).

Methods: From 2012 to 2019 in the First Affiliated Hospital of Dalian Medical University, 44 CRC patients were followed up. We performed immunohistochemical analysis, Western blot and RT-PCR analysis on CRC and adjacent normal tissues for investigating the expression of ACKRs and their relationships to clinicopathologic features and survival rate.

Results: The expression of three ACKRs, CCX-CKR, DARC and D6, in normal colon tissues is higher than that in colorectal cancer tissues. Meanwhile, the expression of corresponding ligands CCL21, CCL2 and CCL22 shows the opposite. Low expression of ACKRs in colon cancer tissues was closely related to the potential of lymph node metastasis $(P=0.001)$. When compared with triple-negative ACKR expression, co-expression of the ACKRs predicted better outcomes of colorectal cancer patients with statistical significance $(P=0.011)$.

Conclusions: The loss of ACKRs may play important roles in lymph node metastasis of CRC. ACKR expression may be considered as prognostic markers in CRC patients.

\section{Background}

In the chemokine system, a group of special receptors, called atypical chemokine receptors (ACKRs) or chemokine decoy receptors, recognize and bind the ligands without causing any signal transduction as they have the ability to capture the chemokines and to annihilated the signals [1-2]. Recently, three ACKRs, Duffy antigen receptor for chemokines (DARC or ACKR1), D6 (ACKR2) and ChemoCentryx chemokine receptor (CCX-CKR or ACKR4), have been widely concerned on their close associations with the occurrence and metastasis of tumors [3].

DARC functions by clearing the angiogenic chemokines intracellularly, by binding CXC (cysteines separated by a single amino acid) chemokines and some CC (cysteines next to each other) chemokines including CCL2, CCL5, CCL11, et al [4, 5]. D6 eliminates almost all inflammatory CC chemokines, such as CCL2, CCL3L1, et al [6]. CCXCKR binds and clears the constitutively expressed chemokines including CC chemokine ligands CCR7/CCLI9 (CCL21), CCR9/CCL25 and CXCR5/CXCLI3 playing an important role in the survival, proliferation and metastasis of tumor [7].

Three ACKRs,CCX-CKR, DARC and D6, are involved in the invasion and metastasis of cervical squamous cell carcinoma [8]. Similarly, loss of ACKRs promotes the development of tumor and co-expression of them is predictive of favorable outcomes in breast cancer [9]. CCX-CKR is a negative predictor of colon cancer metastasis, and it regulates the migration and invasion of tumor cells which is positively correlated with patients' survival [10]. Low D6 expression found in colon adenocarcinomas was correlated to more invasive tumors, but the relevant survival analysis has not been conducted. [11] Nevertheless, the role of DARC in colorectal cancer (CRC) has not been elucidated. We aim to examine the expression of three ACKRs to analyze the correlation between their expression and clinicopathologic characteristics or patient outcomes in colorectal cancer (CRC).

\section{Materials And Methods}




\section{Human colorectal cancer tissue samples}

The present study included 44 patients with colorectal cancer who were followed up after surgery in the First Affiliated Hospital of Dalian Medical University from 2012 to June 2019. No patient had received preoperative chemotherapy or radiotherapy. Out of the 44 tissue samples, 26 included both CRC and matched paracancerous tissues. Specimens were obtained at the time of surgery and were immediately frozen in liquid nitrogen (for western blotting and RT-PCR analysis, the protocols were described in Supplementary materials and the sequences of PCR primers were listed in Supplementary Table S1) and fixed in 10\% neutral-buffered formalin and embedded in paraffin (for immunohistochemistry analysis, IHC). The patients were between 28-89 years old, including 25 males and 19 females, 15 cases with and 29 cases without lymph node metastasis. The study was conducted in accordance with the principles of the Declaration of Helsinki, and the study protocol was approved by the Ethics Committee of First Affiliated Hospital of Dalian Medical University (KY-FB-2019-20). Because of the retrospective nature of the study, patient consent for inclusion was waived.

\section{Immunohistochemistry}

Immunohistochemical staining was performed to detect the expression of DARC, D6, CCX-CKR and corresponding chemokine ligands CCL2, CCL22 and CCL21 in CRC tissues, adjacent normal colorectal tissues and metastatic lymph nodes. The antibody against DARC (Proteintech, 1:100), D6 (Proteintech, 1:100), CCX-CKR (Abcam, 1:150), CCL22 (Abcam, 1:100), CCL2 (Abcam, 1:100), and CCL21 (Abcam, 1:100) were used for antigen detection. Two pathologists evaluated all stained slides separately. For DARC, D6, CCX-CKR, CCL2, CC22 and CCL21, the intensity of staining is semi-quantitatively graded into four levels: - (negative staining); + (weak staining = light yellow); ++ (moderate staining = yellowish brown) and +++ (strong staining = brown) [10].

\section{Statistical analysis}

All statistical analyses were carried out using SPSS (version 17.0, Chicago, USA) statistical software. The cumulative survival rate was calculated as the time of the surgery to time of death or first recurrence. Survival of patients was estimated by Kaplan-Meier analysis and the differences were compared by the log-rank test. The correlation between ACKR expression and clinicopathologic features was assessed using the $\chi 2$ test or Fisher's exact test, while the correlation between ACKR, CCL2, CCL21, CCL22 and the intensity of staining was evaluated using the Spearman's rank. The paired Student's T-test was used to compare the expression of ACKRs and their ligands in tumor tissues and corresponding paracancerous tissues $(n=21)$ analyzed with Western blotting and PCR methods (shown in Supplementary materials). $P<0.05$ in all cases was considered statistically significant.

\section{Results}

\section{Expression of ACKRs in CRC tissues, adjacent normal tissues and lymph nodes metastasis}

To investigate the potential roles of ACKRs in the progression and metastasis of CRC, we performed IHC to analyze the 44 specimens of which 26 included adjacent normal colorectal tissues. The results showed that in normal intestinal glands CCX-CKR, D6 and DARC expressed at a moderate to high level (81, 62 and 73 percent of specimens, respectively, Fig. 1A and Table 1) and in most of the non-metastasis cases $(79,72$ and 83 percent of specimens, respectively, Fig. 1A and Table 2 ACKR+). Further, relatively low expression of ACKRs was found in metastatic CRC specimens and corresponding LN metastasis (Fig. 1B and Table 2). The chemokines, CCL21, CCL22 and CCL2, showed distinctly higher expression in CRC tissues compared with adjacent normal tissues 
(Fig. 1A and Table 1). Frozen specimens were subjected to Western blotting and RT-PCR analysis. Consistent with $\mathrm{IHC}$ results, higher protein level of ACKRs was observed in paracancerous normal tissues than cancer tissues, whereas the expression of corresponding ligands to the three ACKRs showed a reverse trend (Suppl Fig. S1). Similarly, the findings above were proved on transcriptional level (Suppl Fig. S2).

Table 1

Expression of three atypical chemokine receptors and their corresponding ligands in colorectal cancer tissues $(n=44)$ and adjacent normal tissues $(n=26)$.

\begin{tabular}{|lllllll|}
\hline & Tissues & $-(\%)$ & $+(\%)$ & $++(\%)$ & $+++(\%)$ & P \\
\hline CCX-CKR & Cancer & $16(36)$ & $7(16)$ & $11(25)$ & $10(23)$ & 0.002 \\
\hline & Adjacent normal & $0(0)$ & $5(19)$ & $9(35)$ & $12(46)$ & \\
\hline CCL21 & Cancer & $8(18)$ & $10(23)$ & $15(34)$ & $11(25)$ & 0.001 \\
\hline & Adjacent normal & $4(15)$ & $11(42)$ & $6(24)$ & $5(19)$ & \\
\hline D6 & Cancer & $17(38)$ & $12(27)$ & $5(11)$ & $10(23)$ & $<0.001$ \\
& Adjacent normal & $0(0)$ & $10(38)$ & $7(27)$ & $9(35)$ & \\
\hline & Cancer & $5(11)$ & $15(34)$ & $10(23)$ & $14(32)$ & 0.001 \\
\hline DARC & Adjacent normal & $6(24)$ & $10(38)$ & $5(19)$ & $5(19)$ & \\
\hline & Cancer & $16(36)$ & $11(25)$ & $7(16)$ & $10(23)$ & 0.001 \\
\hline CCL2 & Adjacent normal & $0(0)$ & $7(27)$ & $12(46)$ & $7(27)$ & \\
\hline & Cancer & $7(16)$ & $12(27)$ & $11(25)$ & $14(32)$ & 0.001 \\
\hline
\end{tabular}

Table 2

Expression of ACKRs in CRC with or without lymph node (LN) metastasis.

\begin{tabular}{|lllll|}
\hline ACKRs & LN status & \multicolumn{2}{l}{ ACKR expression } & \multirow{2}{*}{ P } \\
\cline { 3 - 4 } & & -+ & \\
\hline CCX-CKR & Metastasis & $10(67 \%)$ & $5(33 \%)$ & 0.003 \\
\hline & Non-metastasis & $6(21 \%)$ & $23(79 \%)$ & \\
\hline D6 & Metastasis & $9(60 \%)$ & $6(40 \%)$ & 0.036 \\
\hline & Non-metastasis & $8(27 \%)$ & $21(72 \%)$ & \\
\hline DARC & Metastasis & $10(67 \%)$ & $5(33 \%)$ & 0.003 \\
\hline
\end{tabular}

The correlation of ACKRs expression with clinicopathological parameters

A significant association of decreased expression of the three ACKRs with lymph node-positive tumors was shown (Table 3). Positive expression of D6 was observed correlated with moderate-to-high grade differentiation $(P=$ 
0.011, Table 3). However, differentiation status of CRC had little impact on patients' survival (Supplementary Fig. S3)

Table 3

Correlation of ACKR expression in patients according to clinicopathologic characteristics $(n=44)$

\begin{tabular}{|c|c|c|c|c|c|c|c|c|c|}
\hline Characteristics & cases & $\begin{array}{l}\text { DARC+ } \\
\text { N (\%) }\end{array}$ & $\mathbf{P}$ & $\begin{array}{l}\text { D6+ N } \\
\text { (\%) }\end{array}$ & $\mathbf{P}$ & $\begin{array}{l}\text { CCX- } \\
\text { CKR+N } \\
(\%)\end{array}$ & $\mathbf{P}$ & $\begin{array}{l}\text { Triple+ } \\
\text { N (\%) }\end{array}$ & $\mathbf{P}$ \\
\hline All CRC & 44 & 28 & & 27 & & 28 & & 15 & \\
\hline Age(years) & & & 0.617 & & 0.723 & & 0.851 & & 0.627 \\
\hline$\leq 60$ & $13(31)$ & $9(69.2)$ & & $9(69.2)$ & & $8(61.5)$ & & $5(38.4)$ & \\
\hline$>60$ & $31(69)$ & 19(61.2) & & $18(58.1)$ & & $20(64.5)$ & & $10(32.2)$ & \\
\hline Gender & & & 0.066 & & 0.198 & & 0.728 & & 0.426 \\
\hline Male & $26(57)$ & $16(61.5)$ & & $18(69.2)$ & & $16(61.5)$ & & $7(26.9)$ & \\
\hline Female & $18(43)$ & $13(72.2)$ & & $9(50)$ & & $12(66.7)$ & & $8(4.4)$ & \\
\hline $\begin{array}{l}\text { Tumor } \\
\text { diameter }\end{array}$ & & & 0.906 & & 0.907 & & 0.772 & & 0.574 \\
\hline$\leq 5 \mathrm{~cm}$ & 28(54) & $18(64.2)$ & & $17(60.7)$ & & $17(60.7)$ & & $9(32.1)$ & \\
\hline$>5 \mathrm{~cm}$ & $16(46)$ & $10(62.5)$ & & $10(62.5)$ & & $9(56.2)$ & & $6(37.5)$ & \\
\hline LN metastasis & & & 0.003 & & 0.036 & & 0.003 & & 0.001 \\
\hline+ & $15(35)$ & $5(33.3)$ & & $6(40)$ & & $5(33.3)$ & & $1(6.7)$ & \\
\hline - & $29(65)$ & 23(79.3) & & $21(72.4)$ & & $23(79.3)$ & & $14(48.2)$ & \\
\hline Differentiation & & & 0.422 & & 0.011 & & 0.170 & & 0.641 \\
\hline Poorly & $9(20.4)$ & $5(55.5)$ & & $2(22.2)$ & & $4(44.4)$ & & $3(33.3)$ & \\
\hline $\mathrm{M}-\mathrm{H}$ & $35(79.5)$ & $23(65.7)$ & & $25(71.4)$ & & $24(68.5)$ & & $12(34.2)$ & \\
\hline
\end{tabular}

\section{Survival analysis}

Kaplan-Meier analysis was used to estimate cancer-specific survival based on IHC results and follow-up data. The number of patients expressing each ACKR was shown in Fig. 2A, including 27 patients with more than one ACKR expression (two or three co-expressing ACKRs) and 4 triple-ACKR-negative cases. The survival analysis indicated that co-expression of two or more ACKRs in CRC evidently linked with prolonged patients' survival when compared with triple-negative cases $(P=0.011$, Fig. $2 B$ ). Neither single ACKR expression nor lymph node status of metastasis has significant correlation with the survival rate (Fig. 2. C-F). Further, we investigated gene-expression related survival in TCGA patient samples with The Human Protein Atlas website (https://www.proteinatlas.org) [12]. Interestingly, low expression of $A C K R 2 / D 6$ or $A C K R 4 / C C X-C K R$, but not that of $A C K R 1 / D A R C$, is associated with poor patient survival in CRC (Supplementary Fig. S4). 


\section{Discussion}

In the current study, we observed that the expression of three ACKRs, CCX-CKR, DARC and D6, in normal colon tissues was higher than that in colorectal cancer tissues; and the expression of corresponding ligands, CCL21, CCL2 and CCL22, showed an inverse trend. Low expression of ACKRs in colon cancer tissues was related to the potential of lymph node metastasis. Co-expression of ACKRs is correlated to better outcomes of colorectal cancer patients.

Published studies have clarified the function of ACKRs in some human cancers [8,9] and some also provide novel molecular targets in cancer therapy [13]. Zhu et al. reported that CCX-CKR alone expression was positively correlated to the patients' survival rate [10], that is in agreement with gene-expression related survival (TCGA, Figure S4). However, for the first time, we observed the co-expression of two or more ACKRs, not single, predicted better outcomes of the CRC patients. The differences between protein- and gene-expression related patients' survival curves (Fig. 2 and Figure S4) are likely due to insufficient cases included which is a limitation of our present study. It is need to be improved with more cases in our future planed study including more than 300 patients.

The work of Zeng et. al. linked the absence of ACKRs to lymph node metastasis of early breast cancers [14]. According to their study the absence of DARC, D6 and CCX-CKR was related to a higher expression of matrix metalloproteinase 9 (MMP-9) and vascular endothelial growth factor (VEGF) [14] which were produced by the tumor cells in response to some CC chemokines (CCL2, CCL19, CCL21 et.al.) [15-17]. In consistent with Zeng's findings, our results proved the loss of ACKRs expression was positively related to lymph node metastasis in CRC patients, which suggested that chemokines and ACKRs are potential therapeutic targets for CRC treatment.

\section{Conclusion}

Our work unveiled the correlation between ACKRs co-expression and better prognosis and preliminary demonstrated the potential roles of ACKRs as a set of combined metastatic markers for CRC patients.

\section{Abbreviations}

ACKR: atypical chemokine receptors; CCX-CKR: ChemoCentryx chemokine receptor; CRC: colorectal cancer; DARC: duffy antigen receptor for chemokines; LN: lymph node; RT-PCR: reverse transcription-polymerase chain reaction; WB: Western blotting

\section{Declarations}

\section{Acknowledgements}

Not applicable.

\section{Authors' contributions}

PL- corresponding author: conception, design and study supervision. PL,HD,YH and CZ: acquisition and analysis of the data, writing the manuscript; HD, YH and CZ: performance of the experiments; TZ,JC, LJ , DL and LK: acquisition of the specimens and the follow-up data; LG and LY : evaluation of the immuno-stained slides. 


\section{Funding}

This work was supported by Natural Science Foundation of Liaoning Province (Liaoning Provincial Natural Science Foundation 2015020296) to Pin Liang.

\section{Availability of data and materials}

All data generated or analyzed during this study are included in this published article.

\section{Ethics approval and consent to participate}

The study was conducted in accordance with the principles of the Declaration of Helsinki, and the study protocol was approved by the Ethics Committee of First Affiliated Hospital of Dalian Medical University (KY-FB-2019-20).

\section{Consent for publication}

Not applicable.

\section{Competing interests}

The authors declare that the research was conducted in the absence of any commercial or financial relationships that could be construed as a potential conflict of interest.

\section{Author details}

${ }^{1}$ Department of Gastrointestinal Surgery, the First Affiliated Hospital, Dalian Medical University, Dalian 116011, China. ${ }^{2}$ Department of Pathophysiology, College of Basic Medical Sciences, Dalian 116044, China. ${ }^{3}$ The First Affiliated Hospital, Anhui Medical University, Hefei 230022, China. ${ }^{4}$ Department of Histology and Embryology, College of Basic Medical Sciences, Dalian116044, China.

\section{References}

1. Segerer S, Jedlicka J, Wüthrich RP. Atypical chemokine receptors in renal inflammation. Nephron Experimental Nephrology. 2010;115:e89-95.

2. Nibbs R, Graham G, Rot A. Chemokines on the move: control by the chemokine "interceptors" Duffy blood group antigen and D6. Semin Immunol. 2003;15:287-94.

3. Bachelerie F, Graham GJ, Locati M, Mantovani A, Murphy PM, Nibbs R, et al. New nomenclature for atypical chemokine receptors. Nat Immunol. 2014;15:207-8.

4. Rot A. Contribution of Duffy antigen to chemokine function. Cytokine Growth Factor Rev. 2005;16:687-94.

5. Kashiwazaki M, Tanaka T, Kanda H, Ebisuno Y, Izawa D, Fukuma N, et al. A high endothelial venule-expressing promiscuous chemokine receptor DARC can bind inflammatory, but not lymphoid, chemokines and is dispensable for lymphocyte homing under physiological conditions. Int Immunol. 2003;15(10):1219-27.

6. Fra AM, Locati M, Otero K, Sironi M, Signorelli P, Massardi ML, et al. Cutting edge: scavenging of inflammatory cc chemokines by the promiscuous putatively silent chemokine receptor D6. J Immunol. 2003;170:2279-82.

7. Haraldsen G, Rot A. Coy decoy with a new ploy: interceptor controls the levels of homeostatic chemokines. Eur J Immunol. 2006;36(7):1659-61. 
8. Hou T, Liang D, Xu L, Huang X, Huang Y, Zhang Y. Atypical chemokine receptors predict lymph node metastasis and prognosis in patients with cervical squamous cell cancer. Gynecol Oncol. 2013;130:181-7.

9. Zeng XH, Ou ZL, Yu KD, Feng LY, Yin WJ, Li J, et al. Coexpression of atypical chemokine binders (ACBs) in breast cancer predicts better outcomes. Breast Cancer Res Treat. 2010;125:715-27.

10. Zhu Y, Tang W, Liu Y, Wang G, Liang Z, Cui L. CCX-CKR expression in colorectal cancer and patient survival. The International Journal of Biological Markers. 2018;29:e40-e8.

11. Langenes V, Svensson H, Börjesson L, Gustavsson B, Bemark M, Sjöling $\AA$, et al. Expression of the chemokine decoy receptor D6 is decreased in colon adenocarcinomas. Cancer Immunol Immunother. 2013;62:1687-95.

12. Uhlen M, Zhang C, Lee S, Sjöstedt E, Fagerberg L, Bidkhori G, et al. A pathology atlas of the human cancer transcriptome. Science. 2017;357:eaan2507.

13. Mollica Poeta V, Massara M, Capucetti A, Bonecchi R. Chemokines and Chemokine Receptors: New Targets for Cancer Immunotherapy. Front Immunol. 2019;10:379.

14. Zeng XH, Ou ZL, Yu KD, Feng LY, Yin WJ, Li J, et al. Absence of multiple atypical chemokine binders (ACBs) and the presence of VEGF and MMP-9 predict axillary lymph node metastasis in early breast carcinomas. Med Oncol. 2014;31:145.

15. Wang J, Ou ZL, Hou YF, Luo JM, Shen ZZ, Ding J, et al. Enhanced expression of Duffy antigen receptor for chemokines by breast cancer cells attenuates growth and metastasis potential. Oncogene. 2006;25(54):7201-11.

16. Wu FY, Ou ZL, Feng LY, Luo JM, Wang LP, Shen ZZ, et al. Chemokine decoy receptor d6 plays a negative role in human breast cancer. Mol Cancer Res. 2008;6(8):1276-88.

17. Feng LY, Ou ZL, Wu FY, Shen ZZ, Shao ZM. Involvement of a Novel Chemokine Decoy Receptor CCX-CKR in Breast Cancer Growth, Metastasis and Patient Survival. Clin Cancer Res. 2009;15(9):2962-70.

\section{Figures}




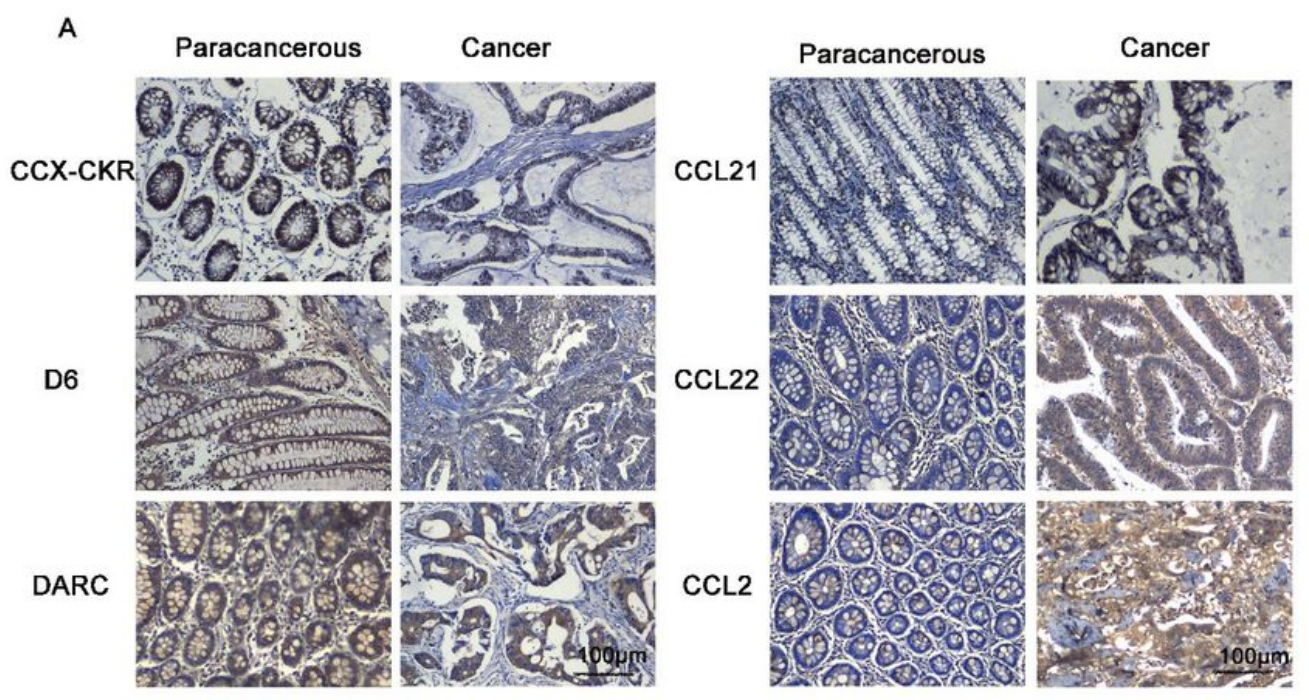

B

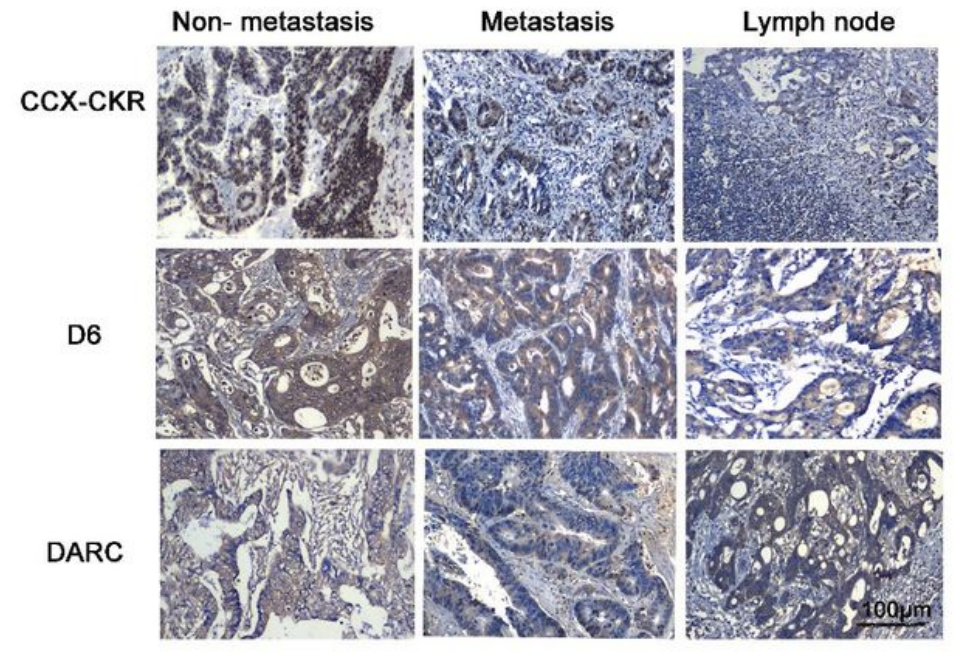

\section{Figure 1}

Representative immunostaining of three atypical chemokine receptors (CCX-CKR, DARC and D6) and corresponding ligands (CCL21, CCL2 and CCL22) expression in tissues of CRC patients. (A) Expression of ACKRs and their ligands in colorectal cancer tissues, as well as in paracancerous normal tissues; (B) Expression of ACKRs in colons with or without lymph node metastasis. Scale bar, $100 \mu \mathrm{m}$. 
A

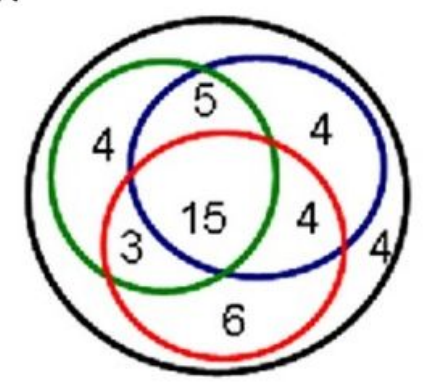

$\mathrm{D} 6+\square \mathrm{CCX}-\mathrm{CKR}+\square \mathrm{DARC}+$

D

DARC $P=0.415$

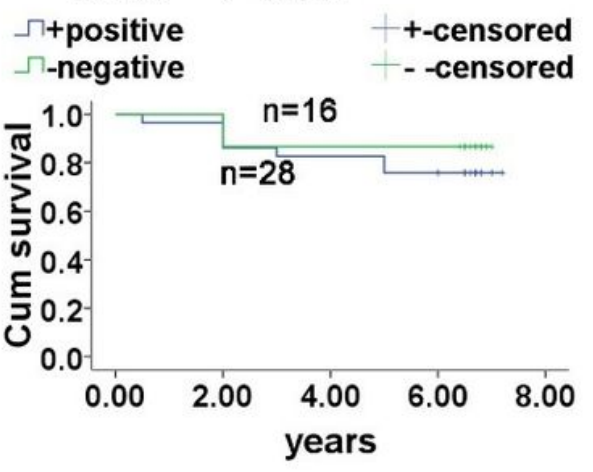

B $\quad P=0.011$

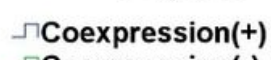

$\neg$ Coexpression(+)

$\neg$ Coexpression(-)

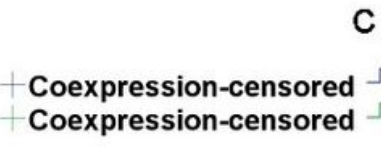

C $\quad \mathrm{P}=0.601$

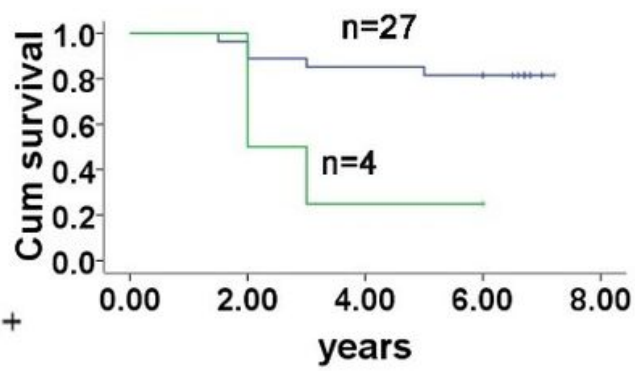

$\neg$ metastasis
$\neg$ non- metastasis

metastasis-censored non- metastasiscensored

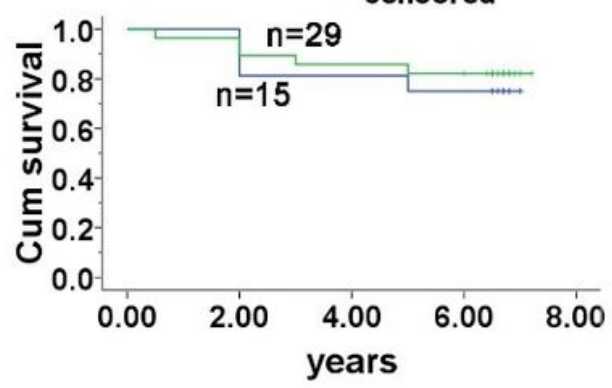

E

\section{CCX-CKR}

$\mathrm{P}=0.259$

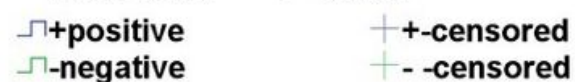

$\neg$-negative

t - -censored

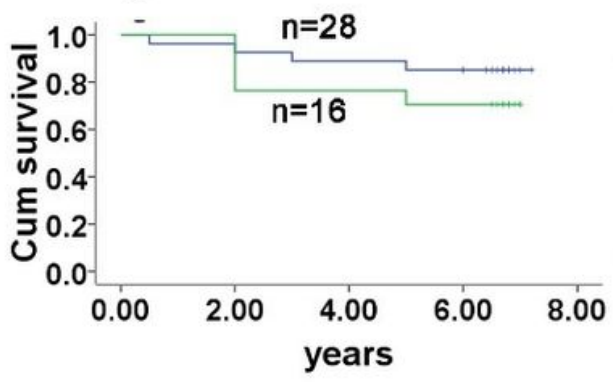

F
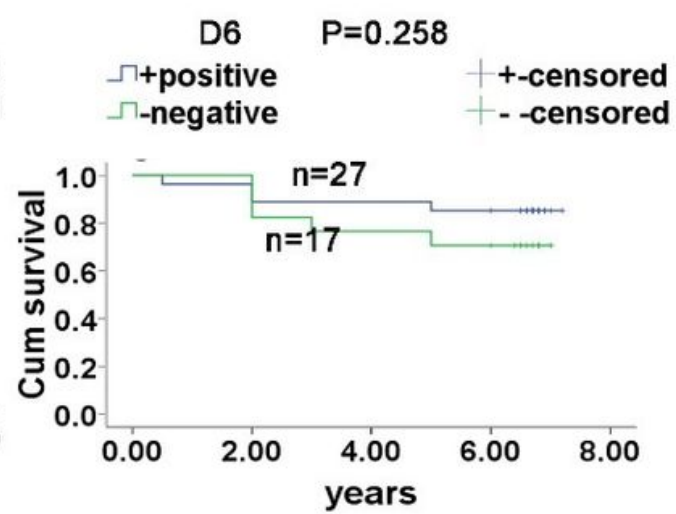

Figure 2

Kaplan-Meier analysis of patients' survival in relation to ACKRs expression and lymph node metastasis in 44 CRC patients. (A) Schematic view of the number of patients according to immuno-reactivity of paraffin-embedded specimens. (B) Cumulative survival curves for co-expression of double- or triple positive ACKR subgroup and triplenegative subgroup. (C) Cumulative survival in relation to lymph node metastasis. (D-F) Cumulative survival curves in relation to DARC (D), CCX-CKR (E) and D6 (F) expression in 44 colorectal cancer patients, respectively.

\section{Supplementary Files}

This is a list of supplementary files associated with this preprint. Click to download.

- FigS1.docx

- Figs2.docx

- FigS3.docx

- FigS4.docx

- Supplementarymaterials.doc 